\title{
Técnica de alisamento por Splines Cúbicas aplicada para suavização dos efeitos dos problemas térmicos inversos com convecção e radiação
}

\author{
Letícia Hiromi Kubo Juliana de Oliveira \\ Departamento de Ciências Biológicas, FCLA, UNESP \\ 19806-900, Assis, SP \\ E-mail: leticiahk@yahoo.com.br_ｊuliana@assis.unesp.br
}

\section{RESUMO}

Sistemas térmicos estudam o comportamento da transferência de calor ao longo do tempo através da diferença de temperatura, respeitando a Primeira Lei da Termodinâmica "A quantidade de energia acumulada deve ser igual a quantidade de energia perdida"[1]. Tais sistemas são amplamente estudados pelas indústrias para o desenvolvimento de equipamentos, como trocadores de calor, evaporadores, aquecedores radiantes, entre outros, com a finalidade de analisar e extrair parâmetros dos processos de transferência de calor sendo os mais estudados condução, convecção e radiação. Uma das áreas que tem sido impulsionada pelas pesquisas industriais que envolvem sistemas térmicos é a de problemas inversos, que estão relacionados com a determinação de causas através da observação de efeitos, porém são intrinsecamente mal condicionados, significando que o processo será extremamente sensível a erros experimentais e numéricos a ponto de comprometer completamente os resultados obtidos.

Desta forma, este projeto tem por objetivo a análise da aplicação da técnica de alisamento por Splines Cúbicas em problemas térmicos inversos a fim de suavizar os efeitos causados pela instabilidade dos problemas inversos na reconstrução da temperatura de um sistema térmico.

A modelagem matemática realizada neste trabalho considera o acúmulo térmico (energia armazenada), a convecção (transferência de calor entre a sonda e o meio) e a radiação (energia emita pelo meio), Equação (1):

$$
\underbrace{M C \frac{d T_{\text {ind }}}{d t}}_{\text {AcúmuloTérmico }}-\underbrace{h A\left(T_{\text {proc }}-T_{\text {ind }}\right)}_{\text {Conveç̧̃̃o }}-\underbrace{\varepsilon \sigma A\left(T_{\infty}^{4}-T_{\text {ind }}^{4}\right)}_{\text {Radiação }}=0
$$

sendo $M(\mathrm{~kg})$ massa do encapsulamento, $C(\mathrm{~J} / \mathrm{kgK})$ calor específico, $A\left(\mathrm{~m}^{2}\right)$ área, $\mathrm{h}\left(\mathrm{W} / \mathrm{m}^{2} \mathrm{~K}\right)$ coeficiente de convecção, $0 \leq \varepsilon \leq 1$ emissividade, $\sigma=5,67 \times 10^{-8}\left(\mathrm{~W} / \mathrm{m}^{2} \mathrm{~K}^{4}\right)$ constante de Stefan-Boltzmann. A metodologia aplicada consiste em considerar dois sensores térmicos inseridos em um sistema reagente. $\mathrm{O}$ primeiro sensor fornece a temperatura real do processo (temperatura de referência), $T_{\text {proc }}$, e o segundo sensor é protegido e fornece a temperatura do escoamento (indicada), $T_{i n d}$, de forma atrasada e atenuada. Portanto, é possível reconstruir a temperatura do processo, $T_{r e c}$, a partir da temperatura indicada pelo sensor, Equação (2):

$T_{\text {proc }, i}=T_{\text {rec }, i}=\frac{\tau}{\Delta t}\left(T_{\text {ind }, i}-T_{\text {ind }, i-1}\right)+T_{\text {ind }, i}-\gamma_{i}\left(T_{\infty}-T_{\text {ind }, i}\right)$

sendo $\tau=M C / h A$ (s) constante de tempo da sonda, $\gamma \cong(4 \varepsilon \sigma / h)\left[\left(T_{\infty}+T_{\text {ind }}\right) / 2\right]^{3}$ coeficiente de radiação e $i$ é o passo no tempo.

Entretanto os problemas inversos apesar de eficientes são intrinsecamente mal condicionados, tornando a reconstrução da temperatura pela Equação (2) inviável, pois pequenos erros de medida, como ruídos, são amplificados corrompendo os resultados. Para solucionar esse problema, são aplicadas técnicas de regularização de sinal [2]. Este projeto implementou o alisamento por Splines Cúbicas como método de regularização de sinal, Equação (3):

$$
L=\lambda \sum_{i=1}^{n}\left(\frac{T_{r e c, i}-T_{r e g, i}}{T_{r e c, i}}\right)^{2}+(1-\lambda) \int_{t_{0}}^{t_{n}}\left(T_{r e g}^{\prime \prime}(t)\right)^{2} d t
$$


onde $T_{\text {reg }}$ é a temperatura já alisada após aplicação do método e $0 \leq \lambda \leq 1$ é o parâmetro que controla a suavidade do alisamento. $\mathrm{O}$ algoritmo proposto foi desenvolvido baseado em [3], [4] e [5], e implementado no Matlab ${ }^{\circledR}$.

Vários testes numéricos foram realizados, e com a finalidade de validar a eficiência do algoritmo testes experimentais foram realizados com sinais de temperatura cedidos pela autora Juliana de Oliveira e adquiridos durante o seu doutorado realizado de 2002 a 2006, no Núcleo de Engenharia Térmica e Fluidos da Escola de Engenharia de São Carlos da Universidade de São Paulo (Netef - EESC - USP - São Carlos). Os resultados de um dos experimentos realizados com ar comprimido são apresentados na Figura 1. Para este experimento os parâmetros de entrada são: $d t=0,0625 \mathrm{~s}, \tau=1,69009 \mathrm{~s}$, $\gamma_{\min }=0,0048, \gamma_{\max }=0,0078$ e $\lambda=0,4$, a imagem exibe as temperaturas $T_{\text {proc }}, T_{\text {ind }}$ (obtidas experimentalmente) e $T_{\text {reg }}$ (obtida pelo alisamento por Splines Cúbicas), sendo possível observar como a $T_{\text {reg }}$ se ajusta a $T_{\text {proc }}$.

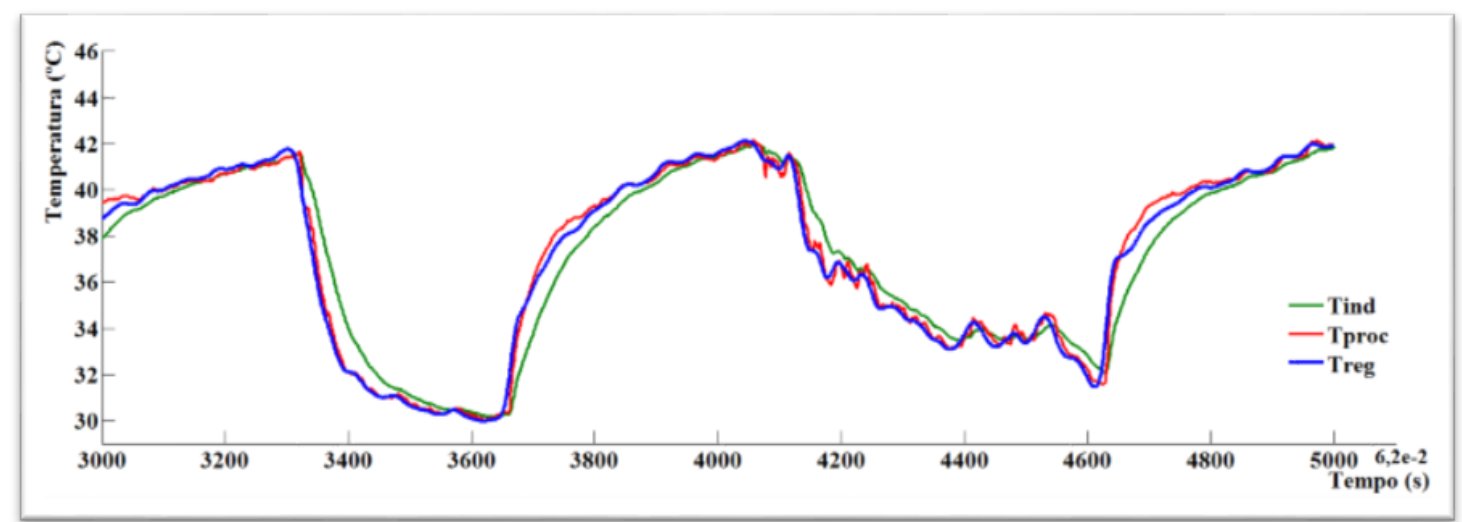

Figura 1. Resultado experimental com ar comprimido

Assim sendo, conclui-se que o alisamento por Splines Cúbicas como método de regularização de sinal é eficiente mesmo com o sistema térmico sofrendo influência da convecção e da radiação e de ruídos embutidos no sinal da temperatura durante a aquisição de dados.

Palavras-chave: problema inverso, splines cúbicas, regularização, convecção e radiação

\section{Referências}

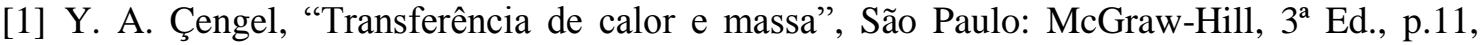
2009.

[2] M. Jakubowska, "Signal Processing in Electrochemistry". Electroanalysis, Vol. 23, p. 553 $572,2011$.

[3] D. S. G. Pollock, "Smoothing with cubic splines", Queen Mary and Westfield College, The University of London, 1993.

[4] C. H. Reinsch, "Smoothing by spline function". Numerische Mathematik, Vol. 10, p.177$183,1967$.

[5] H. L. Weinert, "A fast compact algorithm for cubic spline smoothing", Computational Statistics and Data Analysis, Vol.53, p.932-940, 2009. 